\title{
Protein-losing Enteropathy Caused by Disseminated Mycobacterium Avium Complex Infection in a Patient Receiving Antiretroviral Therapy: An Autopsy Case Report
}

Keiji Konishi ( $\nabla$ k.konishi3117@gmail.com )

Osaka City General Hospital https://orcid.org/0000-0002-1705-6487

Hidenori Nakagawa

Osaka City General Hospital

Akio Nakahira

Osaka City General Hospital

Takahiro Okuno

Osaka City General Hospital

Takeshi Inoue

Osaka City General Hospital

Michinori Shirano

Osaka City General Hospital

\section{Case report}

Keywords: Disseminated Mycobacterium avium complex infection, protein-losing enteropathy, antiretroviral therapy, acquired immunodeficiency syndrome

Posted Date: August 3rd, 2021

DOl: https://doi.org/10.21203/rs.3.rs-754298/v1

License: (c) (i) This work is licensed under a Creative Commons Attribution 4.0 International License. Read Full License

Version of Record: A version of this preprint was published at AIDS Research and Therapy on November 29th, 2021. See the published version at https://doi.org/10.1186/s12981-021-00417-0. 


\section{Abstract}

Background: Disseminated Mycobacterium avium complex infection is an important indicator of acquired immunodeficiency syndrome (AIDS) in patients with advanced human immunodeficiency virus (HIV) infection. Effective antiretroviral therapy has dramatically reduced the incidence and mortality of HIV, although drug resistance and poor medication adherence continue to increase the risk of disseminated $M$. avium complex infection. However, gastrointestinal lesions in cases of disseminated $M$. avium complex infection resulting in protein-losing enteropathy have been rarely discussed. Therefore, we report a case of protein-losing enteropathy caused by disseminated $M$. avium complex infection in a patient undergoing antiretroviral therapy.

Case presentation: A 29-year-old male diagnosed with AIDS for four years and was admitted for a 10month history of refractory diarrhea and fever. Despite receiving antiretroviral therapy, viral load remained elevated due to poor medication adherence. The patient was diagnosed with disseminated M. avium complex infection and started on antimycobacterial drugs two years prior to admission. However, it remained uncontrolled. Previous hospitalization a year before admission was due to hypoalbuminemia and refractory diarrhea. Upper gastrointestinal endoscopy revealed a diagnosis of protein-losing enteropathy caused by intestinal lymphangiectasia, and treatment with intravenous antimycobacterial drugs did not resolve his intestinal lymphangiectasia. The patient inevitably died of sepsis.

Conclusions: Clinical remission is difficult to achieve in patients with AIDS and protein-losing enteropathy caused by disseminated $M$. avium complex infection due to its limited options for parenteral antiretroviral drugs.

\section{Background}

Among nontuberculous mycobacterial infections (e.g., those caused by Mycobacterium avium and $M$. intracellulare), the disseminated $M$. avium complex (DMAC) infection is an important indicator of acquired immunodeficiency syndrome (AIDS) with an advanced human immunodeficiency virus (HIV) infection. Gastrointestinal lesions have been observed in patients with DMAC infections; however, only a few cases of protein-losing enteropathy (PLE) have been reported. Therefore, we present a case of PLE caused by DMAC infection in a patient with an uncontrolled HIV infection caused by poor drug absorption.

\section{Case Presentation}

A 29-year-old man was admitted in August, 2019 for a 10-month history of refractory diarrhea and fever. In September 2015, he was diagnosed with HIV infection and AIDS following the onset of esophageal candidiasis, cytomegalovirus (CMV) enteritis, and Pneumocystis jirovecii pneumonia, which prompted the initiation of antiretroviral therapy (ART) with dolutegravir (DTG), abacavir, and lamivudine. However, viral replication persisted due to poor adherence to medications. In May 2017, the patient was admitted for 
fever with associated cervical and mediastinal lymphadenopathy with elevated viral load (VL) of > 10 million copies/mL. Moreover, his CD4-positive T-lymphocyte count was low (17 cells/ $\mu \mathrm{L}$ ) (Fig. 1 ) and lymph node biopsy revealed $M$. avium. Findings were consistent with a diagnosis of DMAC infection and despite receiving-mycobacterial therapy, such as clarithromycin, ethambutol, and rifabutin, it did not improve his fever and lymphadenopathy. This prompted the initiation of prednisolone for immune reconstitution inflammatory syndrome. In July 2018 , his VL remained uncontrolled due to poor adherence to medications. As a result, the ART was modified to tenofovir disoproxil fumarate (TDF), emtricitabine (FTC), darunavir (DRV), and cobicistat (c).

In October 2018, he presented with watery diarrhea; however, colonoscopy revealed no abnormalities in the lower gastrointestinal tract. In March 2019, the patient also developed optic neuritis due to ethambutol. Because antimicrobial blood cultures remained positive for M. avium, treatment for DMAC infection was continued following the replacement of ethambutol with levofloxacin. However, the patient's fever and watery diarrhea worsened, and blood cultures remained positive for $M$. avium despite treatment. In May 2019, intravenous amikacin was started and later discontinued as the patient had symptoms of vomiting, sensorineural hearing loss, and progressive hypoalbuminemia. A colonoscopy showed an ulcer in the ileocecal region and histopathologic analysis of the biopsy sample confirmed the diagnosis of CMV enteritis. Treatment with intravenous ganciclovir followed by oral maintenance therapy with valganciclovir did not helped with his fever, watery diarrhea, and elevated VL. The HIV drug resistance test detected M184V mutation, which led to further modification of the ART to TDF, FTC, DTG, DRV/c in July 2019. However, VL remained elevated.

In August 2019, the patient was admitted for monitoring of his refractory diarrhea, and a colonoscopy revealed improvement of the ulcers due to $\mathrm{CMV}$ enteritis. A ${ }^{99 \mathrm{~m}} \mathrm{Tc}$ human serum albumin scintigraphy showed a faint accumulation in the center of the left upper quadrant at the third hour, followed by migration at the sixth hour. These findings were consistent with a diagnosis of PLE.

Capsule endoscopy revealed scattered yellowish-white nodules from the horizontal part of the duodenum to the upper jejunum while upper gastrointestinal endoscopy (Fig. 2) showed multiple yellowish-white granular nodules and lymphatic dilatation from the upper duodenal angle to the horizontal part of the duodenum, suggesting that the PLE was due to intestinal lymphangiectasia (IL). Histopathologic analysis showed dense histiocytic infiltration in the mucosal lamina propria, while the Ziehl-Neelsen staining showed numerous acid-fast bacteria. Polymerase chain reaction (PCR) identified the bacteria as $M$. avium, which implicates that DMAC infection is an etiology of IL.

During hospitalization, the blood levels of DTG and DRV were below the detection limits, indicating that the patient did not absorb any of his oral medications due to PLE. As a result, azithromycin, levofloxacin, ganciclovir, and pledonisolone were administered intravenously, and the ART (TDF, FTC, DRV/c) were administered orally. In addition, symptomatic therapy for splenomegaly and vomiting was also received by the patient. 
In February, 2020, he was admitted for worsening pain and hypotension, and as per the request of the patient and his family, cardiopulmonary resuscitation did not occur. He died due to sepsis on the third day after admission.

An autopsy was performed (Fig. 3) and revealed gross pathological findings as follows: (1) diffuse white granular esophageal and duodenojejunal lesions, (2) generalized intestinal edema, (3) enlarged and nodular peritoneal lymph nodes, and (4) enlarged spleen ( $818 \mathrm{~g}$ weight) with similar white granular lesions. Histopathological examination showed acid-fast bacilli in various organs of the body, which includes the esophagus, stomach, duodenum, jejunum, ileum, colon, liver, spleen, lymph nodes (e.g., intestinal, periaortic, mediastinum), lung, adrenal gland, thyroid gland, bone marrow, and renal vessels. In particular, the small intestine had diffuse lesions and showed positive for M. avium complex in PCR. Furthermore, a CMV infection was observed in the colon, lung, and adrenal glands, but the number of infected cells was small. Methicillin-susceptible Staphylococcus aureus was detected in the blood culture and masses in the right lung and bladder mucosa.

\section{Discussion And Conclusions}

We presented a case of PLE due to IL during ART in an adult Japanese man and the etiology of PLE was known to be the obstruction of the intestinal and retroperitoneal lymphatic drainage due to M. avium complex lymphadenitis. As a result of PLE, the patient was unresponsive to oral medications, resulting in poor HIV control which leads to death. However, the prognosis of DMAC infections has improved with the increasing use of ART, and the treatment of rare HIV cases with PLE due to DMAC infection remains challenging $[1,2]$.

DMAC infection is an important opportunistic infection in patients with advanced HIV, especially those with CD4-positive lymphocyte counts of < 50 cells/ $\mu \mathrm{L}$. From 1982 to 1994, the incidence of M. avium complex infections remained high prior to the introduction of highly active antiretroviral therapy (HAART) and prophylactic antibiotics. In the pre-HAART era, M. avium complex infection was a major cause of morbidity and mortality, affecting 15-24\% of patients with AIDS [3], but after the introduction of HAART, the incidence of DMAC infections in HIV patients has decreased significantly. However, due to limited access to health care, resistance to antiretroviral drugs (ARV), and poor medication adherence, he increased risk of $M$. avium complex infection continues [4].

M. avium complex is transmitted via ingestion or inhalation, leading to colonization within the gastrointestinal or respiratory tract. Although colonization may or may not result in infection, these two processes often co-exist. The symptoms of DMAC are non-specific and may mimic other gastrointestinalrelated illnesses. Therefore, isolation of the organism from tissues (e.g., blood, lymph nodes, liver, or bone marrow) is usually required to establish a diagnosis. Upper gastrointestinal endoscopy is an important diagnostic tool that should be considered in HIV-infected individuals with gastrointestinal symptoms if DMAC infection is suspected [5]. According to a report of endoscopic findings in DMAC infection, patients not treated with HAART were more likely to have endoscopic abnormalities than those treated with 
HAART [6]. Endoscopic findings include the following: (1) raised yellow or white plaques and nodules, (2) edema, (3) erosions and ulcers, (4) friability, and (5) decreased mucosal vascular pattern [6]. In the gastrointestinal tract, lesions in the duodenum (76\%) were the most common, followed by the rectum (24\%), ileum (6\%), colon (4\%), jejunum (2\%), and stomach (2\%) [6]. In this case, there were diffuse lesions in the small intestine consistent with PLE due to IL. To the best of our knowledge, $M$. avium complex infections with extensive gastrointestinal tract involved with receiving ART are rarely reported. This study described a rare case of DMAC in an HIV patient due to IL-induced PLE and poor medication adherence.

$\mathrm{IL}$ is a rare condition characterized by an obstruction of lymph vessels supplying the small intestines, potentially causing PLE, and classified into two types: primary and secondary. Primary IL usually occurs in children and adolescents due to an inherent lymphatic anomaly. In contrast, secondary IL occurs in adults due to an underlying disease (e.g., lymphoma, systemic lupus erythematosus, intestinal tuberculosis, retroperitoneal fibrosis, liver cirrhosis, constrictive pericarditis, and abdominal surgery). In our case, the patient had an IL secondary to $M$. avium complex infection lymphadenitis, causing an obstruction of the mesenteric and retroperitoneal lymphatic drainage. PLE may further aggravate the immunodeficiency due to the loss of T-lymphocytes and immunoglobulins (especially IgG) [7]. Therefore, the therapeutic management of PLE is necessary for patients infected with HIV. In the presence of secondary IL, treatment of the primary disease must be continued simultaneously. In our patient, antimycobacterial treatment was more aggressive. In addition to oral rifabutin and ethambutol, we also administered intravenous levofloxacin, azithromycin, and amikacin. However, despite this treatment, the infection persisted.

HIV patients with malabsorption are difficult to control. In some reports, the effective ART regimen for HIV patients with malabsorption was determined using therapeutic drug monitoring (TDM) [8]. In our case, TDM of DTG and DRV was performed, which showed low levels of these drugs in the blood. Currently, all drugs used in ART listed in the guidelines are given orally [9]. Effective ART drugs for HIV patients with malabsorption are limited. In March 2020, the world's first long-acting intramuscular formulation of cabotegravir (CAB) and rilpivirine (RPV) was approved in Canada, making the use of parenteral drugs possible. These drugs had sufficient blood levels with good antiretroviral efficacy when administered once every 1 to 2 months [10-12]. However, the intramuscular formulation in Canada is approved only for maintenance therapy in patients who have been successfully treated. Although parenteral drugs could have been the best option for the patient in our case, the intramuscular formulation is not approved for initial therapy.

We have described a rare case of DMAC due to IL-induced PLE and poor medication adherence in a Japanese patient with HIV. The emergence of drug-resistant viruses prompted further studies to determine the best therapeutic option for HIV patients with PLE in terms of suitability, safety, and efficacy.

\section{Abbreviations}

DMAC: disseminated Mycobacterium avium complex 
AIDS: autoimmune deficiency syndrome

HIV: human immunodeficiency virus

PLE: protein-losing enteropathy

CMV: cytomegalovirus

ART: antiretroviral therapy

DTG: dolutegravir

VL: viral load

TDF: tenofovir disoproxil fumarate

FTC: emtricitabine

DRV: darunavir

c: cobicistat

IL: intestinal lymphangiectasia

PCR: polymerase chain reaction

HAART: highly active antiretroviral therapy

\section{Declarations}

\section{Ethics approval and consent to participate}

Informed consent and protocols was obtained and approved by the Human Ethics Committee of Osaka City General Hospital.

\section{Consent for publication}

Not applicable.

\section{Availability of data and materials}

The datasets used and analyzed during the current study are available from the corresponding author upon reasonable request.

\section{Competing interests}

The authors declare that they have no competing interests. 


\section{Funding}

Not applicable.

\section{Author's contributions}

All authors analyzed and interpreted the patient data. $\mathrm{KK}$ and $\mathrm{HN}$ were major contributors in writing the manuscript. KK and HN were the first co-authors. All authors read and approved the final manuscript.

\section{Acknowledgements}

We appreciate Von Adrian for revising the grammar errors in the manuscript.

\section{References}

1. Ogawa E, Murata M, Unno M, Otaguro S, Kainuma M, Sawayama $Y$, et al. Protein-losing enteropathy during highly active antiretroviral therapy in a patient with AIDS-related disseminated mycobacterial avium complex infection. J Infect Chemother. 2009;15(4):252-6. https://doi.org/10.1007/s10156009-0698-4.

2. Bansal R, Ayoub M, Policar M. Gastrointestinal mycobacterium avium complex. Am J Med Sci. 2021;361(3):e31-e2. https://doi.org/10.1016/j.amjms.2020.09.007.

3. Horsburgh CR. Jr. Mycobacterium avium complex infection in the acquired immunodeficiency syndrome. N Engl J Med. 1991;324(19):1332-8. https://doi.org/10.1056/nejm199105093241906.

4. Palella FJ Jr, Delaney KM, Moorman AC, Loveless MO, Fuhrer J, Satten GA, et al. Declining morbidity and mortality among patients with advanced human immunodeficiency virus infection. HIV Outpatient Study Investigators. N Engl J Med. 1998;338(13):853-60. https://doi.org/10.1056/nejm199803263381301.

5. Werneck-Silva AL, Prado IB. Role of upper endoscopy in diagnosing opportunistic infections in human immunodeficiency virus-infected patients. World J Gastroenterol. 2009;15(9):1050-6. https://doi.org/10.3748/wjg.15.1050.

6. Sun HY, Chen MY, Wu MS, Hsieh SM, Fang CT, Hung CC, et al. Endoscopic appearance of GI mycobacteriosis caused by the mycobacterium avium complex in a patient with AIDS: case report and review. Gastrointest Endosc. 2005;61(6):775-9. https://doi.org/10.1016/s0016-5107(04)027865.

7. Fuss IJ, Strober W, Cuccherini BA, Pearlstein GR, Bossuyt X, Brown M, et al. Intestinal lymphangiectasia, a disease characterized by selective loss of naive CD45RA + lymphocytes into the gastrointestinal tract. Eur J Immunol. 1998;28(12):4275-85. https://doi.org/10.1002/(sici)15214141(199812)28:12<4275::Aid-immu4275>3.0.Co;2-p.

8. Ikuma M, Watanabe D, Yagura H, Ashida M, Takahashi M, Shibata M, et al. Therapeutic Drug Monitoring of Anti-human Immunodeficiency Virus Drugs in a Patient with Short Bowel Syndrome. Intern Med. 2016;55(20):3059-63. https://doi.org/10.2169/internalmedicine.55.6777. 
9. Guidelines for the use of antiretroviral agents in HIV-1 - infected adults and adolescents. Department of Health and Human Services. https://aidsinfo.nih.gov/. Accessed 15 July 2021.

10. Swindells S, Andrade-Villanueva JF, Richmond GJ, Rizzardini G, Baumgarten A, Masiá M, et al. Longacting cabotegravir and rilpivirine for maintenance of HIV-1 suppression. N Engl J Med. 2020;382(12):1112-23. https://doi.org/10.1056/NEJMoa1904398.

11. Overton ET, Richmond G, Rizzardini G, Jaeger H, Orrell C, Nagimova F, et al. Long-acting cabotegravir and rilpivirine dosed every 2 months in adults with HIV-1 infection (ATLAS-2M), 48-week results: a randomized, multicentre, open-label, phase 3b, non-inferiority study. Lancet. 2021;396(10267):19942005. https://doi.org/10.1016/s0140-6736(20)32666-0.

12. Orkin $C$, Oka $S$, Philibert $P$, Brinson $C$, Bassa A, Gusev D, et al. Long-acting cabotegravir plus rilpivirine for treatment in adults with HIV-1 infection: 96-week results of the randomized, open-label, phase 3 FLAIR study. Lancet HIV. 2021;8(4):e185-e96. https://doi.org/10.1016/s2352-3018(20)30340-4.

\section{Figures}

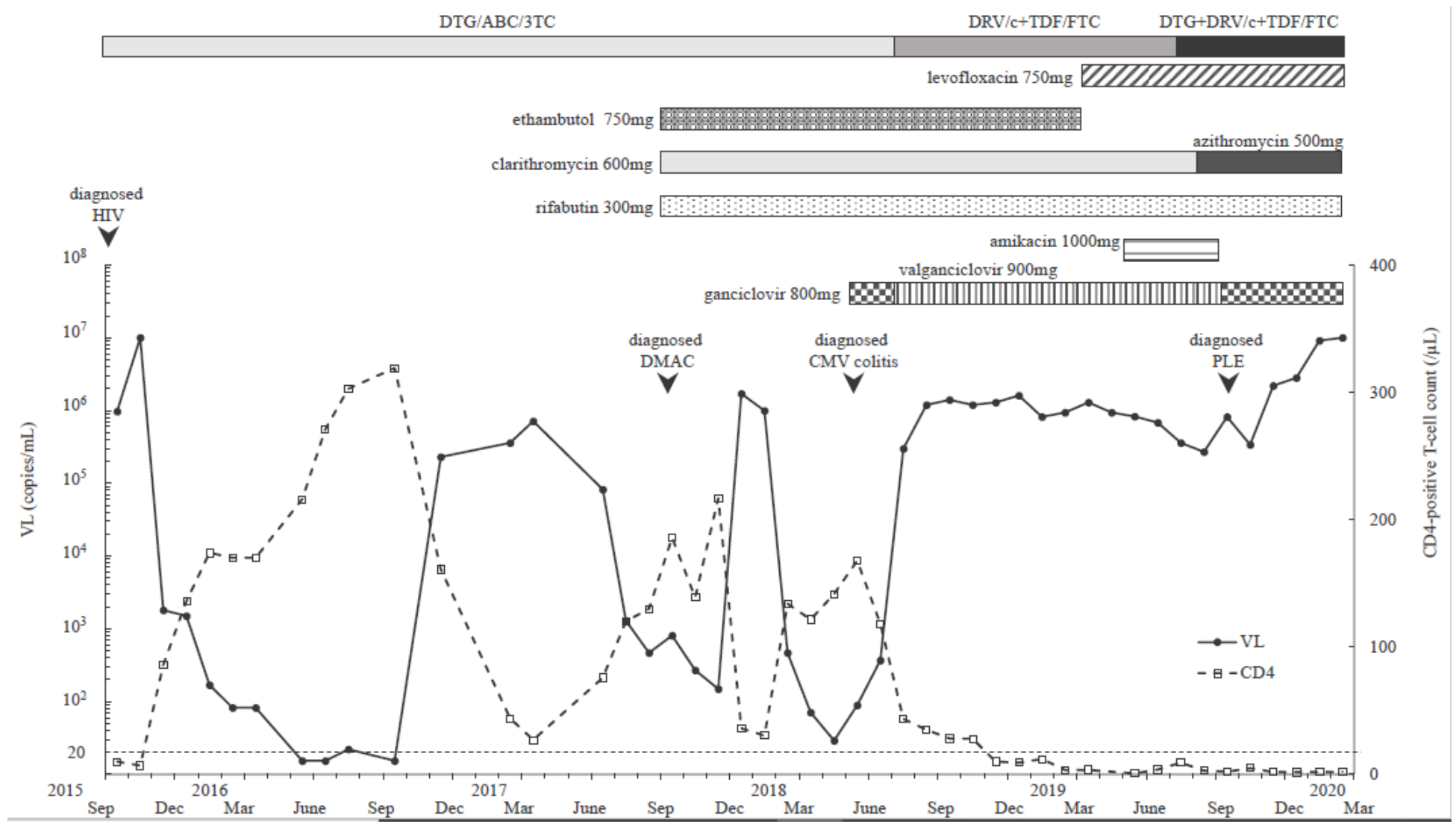

\section{Figure 1}

Time course of therapeutic drug and serial changes in the CD4 cell count and HIV-1 RNA level (VL) in our patient with HIV-1 In the top table, the administered therapeutic drugs. In the bottom table, the VL and CD4 cell count are indicated by open squares and closed circles, respectively. HIV: human immunodeficiency virus, DMAC: disseminated Mycobacterium avium complex, CMV: cytomegalovirus, 
PLE: protein-losing enteropathy, VL: viral load, DTG: dolutegravir, ABC: abacavir, 3TC: lamivudine, DRV: darunavir, c: cobicistat, TDF: tenofovir disoproxil fumarate, FTC: emtricitabine.

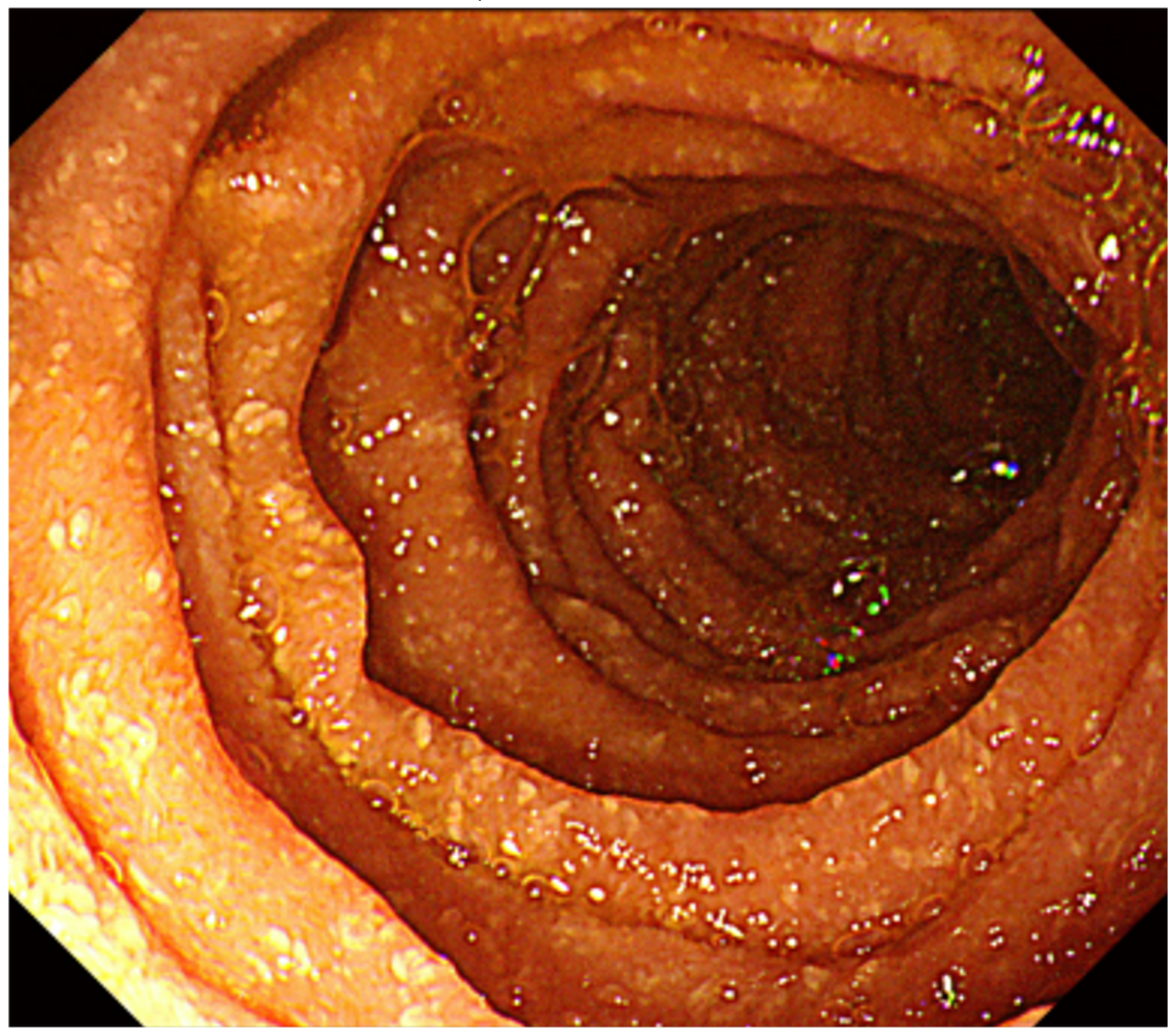

Figure 2

Findings of upper gastrointestinal endoscopy Upper gastrointestinal endoscopy showed multiple yellowish-white granular nodules and lymphatic dilatation from the upper duodenal angle to the horizontal part of the duodenum. 

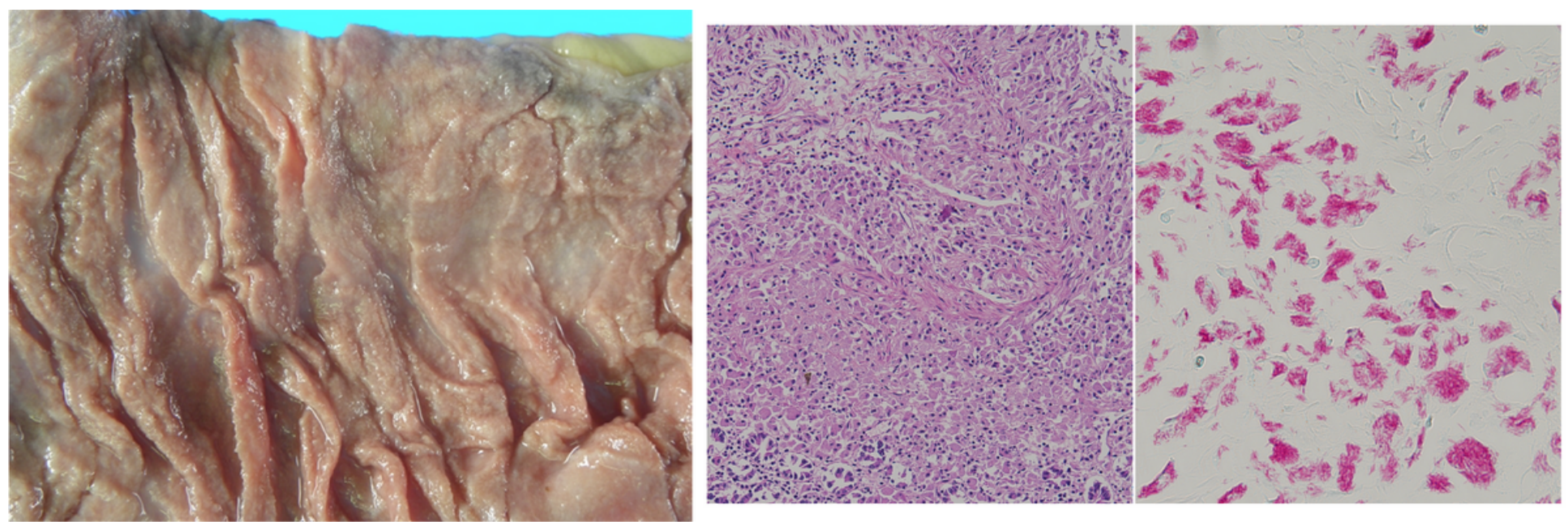

\section{Figure 3}

Gross pathologic findings and histopathologic examination Panel a. Gross pathologic findings showed white granular lesions were diffusely present from the duodenum to the jejunum, and vasodilation was observed. Panel b. Histopathologic analysis showed dense histiocytic infiltration in the mucosal lamina propria, and the Ziehl-Neelsen staining showed numerous acid-fast bacilli (left, hematoxylin and eosin staining; right, Ziehl-Neelsen staining, both magnification $\times 200$ ). 